\title{
Reflections of regular maps and Riemann surfaces
}

\section{Adnan Melekoğlu and David Singerman}

\begin{abstract}
A compact Riemann surface of genus $g$ is called an M-surface if it admits an anti-conformal involution that fixes $g+1$ simple closed curves, the maximum number by Harnack's Theorem. Underlying every map on an orientable surface there is a Riemann surface and so the conclusions of Harnack's theorem still apply. Here we show that for each genus $g>1$ there is a unique M-surface of genus $g$ that underlies a regular map, and we prove a similar result for Riemann surfaces admitting anti-conformal involutions that fix $g$ curves.
\end{abstract}

\section{Introduction}

A regular map is said to be reflexible if it admits a reflection, that is, an orientation-reversing automorphism of order two that has a non-empty fixedpoint set. (Maps and regular maps are described in Section 4.) There is much work in the literature on the question of whether a given regular map is reflexible, (see [8], chapter 8) but there is little work related to the nature of the fixed-point set of a reflection of a regular map. Underlying every map on a surface there is a Riemann surface, which is defined in such a way that every automorphism of the map is a conformal automorphism of the corresponding Riemann surface. Thus when we study reflexible maps we should consider Riemann surfaces that admit an anti-conformal involution. Following a tradition that goes back to Klein [13], we call such transformations symmetries. Whereas compact Riemann surfaces correspond to complex algebraic curves, the symmetric Riemann surfaces correspond to real algebraic curves, the fixed-point set of the symmetry being the real circuits of the curve.

2000 Mathematics Subject Classification: 05C10, 30F10.

Keywords: Regular map, Riemann surface, Platonic surface, M-surface, (M-1)-surface. 
By a classical theorem of Harnack the fixed-point set of a symmetry of a compact Riemann surface of genus $g>1$ consists of at most $g+1$ simple closed curves, or mirrors as we shall call them. A Riemann surface of genus $g$ that admits a symmetry with $g+1$ mirrors is called an $M$ surface, and if it admits a symmetry with $g$ mirrors then it is called an (M-1)-surface. A Riemann surface that underlies a regular map is called a Platonic surface. In this case, by Belyı's theorem the corresponding algebraic curve is defined over the field of algebraic numbers. The main results of this paper will uniquely characterise the Platonic M-surfaces and the Platonic (M-1)-surfaces. These turn out to be Riemann surfaces that are already well-known from the study of Riemann surfaces with large automorphism groups and are the Accola-Maclachlan surfaces, (in the case of M-surfaces) and the Wiman surfaces of type II (in the case of (M-1)-surfaces.) In this way, we find the reflexible maps that admit reflections fixing $g+1$ or $g$ closed curves.

\section{Non-Euclidean Crystallographic Groups}

A discrete group of sense-preserving isometries of the hyperbolic plane $\mathcal{U}$ is called a Fuchsian group. A discrete group of isometries of the hyperbolic plane which might include sense-reversing isometries is called a nonEuclidean crystallographic (NEC) group. An NEC group which is not Fuchsian is called a proper NEC group. The subgroup of an NEC group $\Gamma$ consisting of orientation-preserving transformations is called the canonical Fuchsian group of $\Gamma$ and it is denoted by $\Gamma^{+}$. All NEC groups $\Gamma$ in this paper will be assumed to be cocompact, (which means that the quotient space $\mathcal{U} / \Gamma$ is compact.) If $X$ is a Riemann surface of genus $g>1$ then it can be expressed in the form $\mathcal{U} / K$, where $K$ is a torsion-free Fuchsian group. Any group $G$ of conformal homeomorphisms of $X$ (that may include sensereversing automorphisms) may then be lifted to an NEC group $\Gamma$ acting on $\mathcal{U}$, while if $G$ only contains sense-preserving automorphisms then $\Gamma$ is Fuchsian. In either case there is a smooth homomorphism $\theta: \Gamma \rightarrow G$ whose kernel is $K$, where by smooth we mean that $\theta$ preserves the orders of elements of finite order; equivalently $K$ is torsion-free. The structure of NEC groups is described in [3], [17] and [24]. For completeness we describe the structure of such groups. The signature of an NEC group is defined to be

$$
\left(g ; \pm ;\left[m_{1}, m_{2}, \ldots, m_{r}\right] ;\left\{\left(n_{11}, \ldots, n_{1 s_{1}}\right), \ldots,\left(n_{k 1}, \ldots, n_{k s_{k}}\right)\right\}\right) .
$$

The algebraic and geometric structure of an NEC group is completely determined by its signature. If $\Delta$ is an NEC group with signature (2.1), then $\mathcal{U} / \Delta$ is a compact surface of genus $g$ with $k$ holes. The surface is orientable if + 
sign is used and non-orientable if - sign is used. The integers $m_{1}, m_{2}, \ldots, m_{r}$ are called the proper periods and represent the branching over interior points of $\mathcal{U} / \Delta$ in the natural projection from $\mathcal{U}$ to $\mathcal{U} / \Delta$. The brackets $\left(n_{i 1}, \ldots, n_{i s_{i}}\right)$ are the period cycles and the integers $n_{i 1}, \ldots, n_{i s_{i}}$ are called the link periods and they represent the branching around the $i$ th hole. Now let us describe the presentation of a group with signature (2.1). If the + sign is used, it has generators

(i) $x_{1}, \ldots, x_{r}$ (elliptic elements),

(ii) $c_{10}, \ldots, c_{1 s_{1}}, \ldots, c_{k 0}, \ldots, c_{k s_{k}}$ (reflections),

(iii) $e_{1}, \ldots, e_{k}$ (usually hyperbolic elements but sometimes elliptic),

(iv) $a_{1}, b_{1}, \ldots, a_{g}, b_{g}$ (hyperbolic elements),

and relations

(a) $x_{i}^{m_{i}}=1$, for $i=1, \ldots, r$,

(b) $c_{i, j-1}^{2}=c_{i j}^{2}=\left(c_{i, j-1} c_{i j}\right)^{n_{i j}}=1$, for $i=1, \ldots, k$ and $j=1, \ldots, s_{i}$,

(c) $e_{i}^{-1} c_{i 0} e_{i}=c_{i s_{i}}$ for $i=1, \ldots, k$,

(d) $x_{1} x_{2} \ldots x_{r} e_{1} e_{2} \ldots e_{k} a_{1} b_{1} a_{1}^{-1} b_{1}^{-1} \ldots a_{g} b_{g} a_{g}^{-1} b_{g}^{-1}=1$.

If there is - sign in the signature we replace (iv) by

(iv) $a_{1}, \ldots, a_{g}$ (glide reflections), and (d) by

(d) $x_{1} x_{2} \ldots x_{r} e_{1} e_{2} \ldots e_{k} a_{1}^{2} a_{2}^{2} \ldots a_{g}^{2}=1$.

In this paper we shall often be considering triangle groups. An NEC triangle group is a group of signature $(0 ;+;[] ;\{(l, m, n)\})$ which we say is the NEC triangle group $\Gamma^{*}(l, m, n)$. It is the group generated by the reflections in the sides of a triangle with angles $\pi / l, \pi / m$ and $\pi / n$. The subgroup of index two of this group consisting of orientation-preserving isometries has signature $(0 ;+;[l, m, n] ;\{\})$ is the Fuchsian triangle group $\Gamma[l, m, n]$. We will need some results on inclusions of Fuchsian and NEC groups.

Lemma 2.1 [24] If $l, m, n$ are three distinct integers $\geq 2$ then the only $N E C$ group that contains $\Gamma[l, m, n]$ is $\Gamma^{*}(l, m, n)$. If two or more of $l, m, n$ are equal (say $l=m$ ) then also the $N E C$ group of signature $(0 ;+;[m] ;\{(n)\})$ contains $\Gamma[m, m, n]$. In both cases the index of the inclusion is equal to 2.

Lemma $2.2[22] \Gamma[m, m, n]<\Gamma[2, m, 2 n]$ with index 2 . 


\section{Symmetries of Riemann Surfaces}

An automorphism of a Riemann surface $X$ is a conformal or anti-conformal homeomorphism of $X$ onto itself. The automorphisms of $X$ form a group under the composition of maps which is denoted by Aut $X$ and the subgroup of index one or two consisting of conformal automorphisms is denoted by Aut ${ }^{+}$.

A Riemann surface $X$ is called symmetric if it admits an anti-conformal involution $T: X \rightarrow X$ and such an involution is called a symmetry. If $T$ has no fixed-point, then it is called an antipodal symmetry, otherwise it is called a reflective symmetry. The fixed-point set of $T$ consists of disjoint simple closed curves on $X$ and these curves are called the mirrors of $T$.

The basic results concerning symmetries of compact Riemann surfaces go back to Harnack [9] in 1876 and a useful account of the 19th century work on the subject may be found in part III of Klein's classic book "On Riemann's theory of algebraic functions and their integrals" [13]. Let $X$ be a compact Riemann surface of genus $g$ and $T: X \rightarrow X$ be a symmetry. Thus, $T$ is anti-conformal and $T^{2}=I$. Let $\langle T\rangle$ denote the cyclic group of order two generated by $T$ and $F(T)$ the fixed-point set of $T$. The classical results may be stated as follows.

Theorem $3.1 \quad(i) F(T)$ consists of $k$ mirrors, where $0 \leq k \leq g+1$.

(ii) $X-F(T)$ consists of one component if $X /\langle T\rangle$ is non-orientable and two components if $X /\langle T\rangle$ is orientable.

(iii) If $X-F(T)$ consists of two components, then $g-k$ is odd.

Let $X$ be Riemann surface of genus $g>1$ and $T: X \rightarrow X$ a symmetry of $X$. If $T$ has $g+1$ mirrors, then it is called an $M$-symmetry, and in this case $X$ is called an $M$-surface. Similarly, if $T$ has $g$ mirrors, then it is called an $(M-1)$-symmetry, and $X$ is called an (M-1)-surface. A Riemann surface $X$ is called hyperelliptic if it admits a conformal involution $J$ such that $X /\langle J\rangle$ has genus 0. Equivalently, $J$ has $2 g+2$ fixed-points.

We now give the following theorems which appear in Natanzon [19], [20] and [21], and also Bujalance and Costa [5].

Theorem 3.2 Let $X$ be an $M$-surface. If $X$ is non-hyperelliptic then

(i) $X$ admits exactly one $M$-symmetry,

(ii) Aut $X=C_{2} \times A u t^{+} X$, where $C_{2}$ is generated by the $M$-symmetry and Aut ${ }^{+} X$ is isomorphic to a finite subgroup of the group of isometries of the sphere.

If $X$ is hyperelliptic then $X$ admits exactly two $M$-symmetries and their product is the hyperelliptic involution. 
Theorem 3.3 Let $X$ be an (M-1)-surface of genus $g>2$. If $X$ is nonhyperelliptic then

(i) $X$ admits exactly one (M-1)-symmetry,

(ii) Aut $X=C_{2} \times$ Aut $^{+} X$, where $C_{2}$ is generated by the (M-1)-symmetry and $A u t^{+} X$ is isomorphic to a finite subgroup of the group of isometries of the sphere.

If $X$ is hyperelliptic then $X$ admits exactly two (M-1)-symmetries and their product is the hyperelliptic involution.

We shall also need the following concept.

Definition 3.1 Let $\Gamma$ be an NEC group and $G$ a finite group. A homomorphism $\theta: \Gamma \rightarrow G$ is called admissible if the kernel of $\theta$ is a torsion-free Fuchsian group $K$ with compact quotient space.

Then $X=\mathcal{U} / K$ is a Riemann surface of genus $g$ and $G$ is a subgroup of AutX. The following properties of admissible homomorphisms are given in $[4]$ :

(i) If $g \in \Gamma$ has order $n$, then $\theta(g)$ has order $n$ in $G$,

(ii) $g \in \Gamma^{+}$if and only if $\theta(g) \in A u t^{+} X$,

(iii) If $c \in \Gamma$ is a reflection, then $\theta(c)$ is a reflective symmetry.

(Note that being admissible is stronger than just being smooth as we require the kernel to consist of orientation-preserving isometries).

\section{Maps and Regular maps}

Definition 4.1 A map $\mathcal{M}$ is an embedding of a finite graph $\mathcal{G}$ into a surface $X$ such that the components of $X-\mathcal{G}$, which are called faces of $\mathcal{M}$, are polygonal 2-cells.

We shall require $X$ to be compact, connected, orientable and without boundary. Also we shall require that every edge of $\mathcal{M}$ has one or two vertices. The genus of $\mathcal{M}$ is defined to be the genus of the underlying surface $X$.

We define a dart to be a pair, consisting of a vertex $v$ and an edge directed towards $v$. An edge may be homeomorphic to the closed interval $[0,1]$ or to a circle $S^{1}$ and in the latter case it is called a loop. In the first case it is usual for the edge to have two vertices and two darts. However, we sometimes require the edge only to have just one vertex and one dart as illustrated as $c$ in Figure 1. Such an edge is called a free edge. (For more details see [12].) In this paper our maps will not contain loops and except in one important case, not contain free edges. Since $X$ has no boundary, each 
dart has two sides which are called blades. In Figure 1, $a$ is an edge with two darts, $b$ is a blade, $c$ is a free edge and $d$ is a loop.

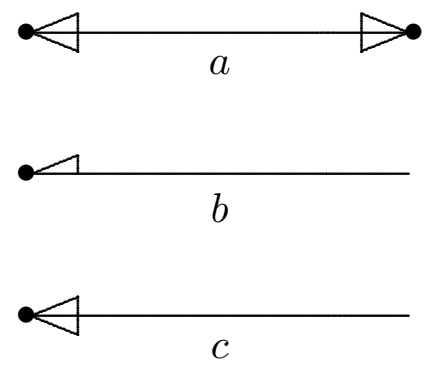

Figure 1

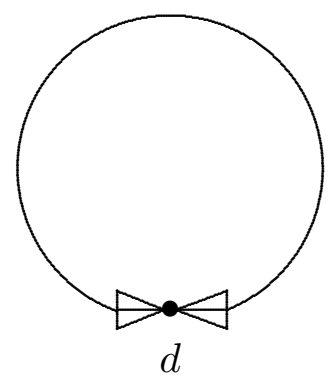

Let $X$ be a Riemann surface of genus $g>1$ and $\mathcal{M}$ be a map on $X$. An automorphism of $\mathcal{M}$ is a homeomorphism of $X$ which leaves the map invariant and preserves incidence and every such automorphism also gives an automorphism of the Riemann surface $X$, see [12]. The automorphisms of $\mathcal{M}$ form a group, called the automorphism group of $\mathcal{M}$, which we shall denote by $A u t \mathcal{M}$. We shall denote the subgroup of $A u t \mathcal{M}$ consisting of orientation-preserving elements by $A u t^{+} \mathcal{M}$. We say that $\mathcal{M}$ is regular if $A u t^{+} \mathcal{M}$ is transitive on the darts. If $\mathcal{M}$ admits an involution $C$ which fixes the mid-point of an edge and interchanges the two darts of the edge without interchanging the two neighbouring faces, then $\mathcal{M}$ is called reflexible and $C$ is called a reflection. It is easy to see that $\mathcal{M}$ is reflexible if and only if Aut $\mathcal{M}$ is transitive on the blades. In general a map is said to be of type $\{m, n\}$ if $m$ is the least common multiple of the vertex valencies and $n$ is the least common multiple of the face sizes, so that a regular map of type $\{m, n\}$ has all vertex valencies equal to $m$ and all face sizes equal to $n$.

The automorphism group of a reflexible regular map $\mathcal{M}$ of type $\{m, n\}$ gives a group of automorphisms of the underlying surface $X$ that lifts to an NEC triangle group of signature $(0 ;+;[] ;\{(2, m, n)\})$ with presentation

$$
\left\langle a, b, c \mid a^{2}=b^{2}=c^{2}=(a b)^{2}=(b c)^{m}=(c a)^{n}=1\right\rangle
$$

and $A u t \mathcal{M}$ is a smooth homomorphic image of this group. If this homomorphism is given by $a \mapsto A, b \mapsto B, c \mapsto C$ then the group of conformal automorphisms of $\mathcal{M}, A u t^{+} \mathcal{M}$, can be generated by $B C=R$ and $C A=S$ which obey

$$
R^{m}=S^{n}=(R S)^{2}=I,
$$

where $R$ is an anticlockwise rotation about a vertex and $S$ is an anticlockwise rotation about a face-centre.

In [12] it was shown that if $\mathcal{M}$ is a map of type $\{m, n\}$ on a Riemann surface $X$, then $X$ is uniformized by a subgroup $M$ of a triangle group $\Gamma$ 
with signature $(0 ;+;[2, m, n] ;\{\})$. The relationship between $\mathcal{M}$ and $M$ is as follows. On $\mathcal{U}$ there lies the universal tessellation $\hat{\mathcal{M}}$ of type $\{m, n\}$ in which every vertex has valency $m$ and every face is a regular $n$-gon. The group $\Gamma$ acts on $\hat{\mathcal{M}}$ as a group of automorphisms, and hence so does $M$. We then have $\hat{\mathcal{M}} / M$ is a map on the Riemann surface $\mathcal{U} / M$ which is isomorphic to $\mathcal{M}$. Moreover, $\mathcal{M}$ is regular if and only if $M \triangleleft \Gamma$. We call $M$ the map subgroup of $\mathcal{M}$.

Definition 4.2 A compact Riemann surface $X$ is called Platonic if $X=$ $\mathcal{U} / K$, where $K$ is a normal subgroup of a triangle group with signature $(0 ;+;[2, m, n] ;\{\})$.

In future work we shall undertake a more detailed study of Platonic surfaces. For now, we just note that a Riemann surface that underlies a regular map is Platonic as are many well-known families of Riemann surfaces such as those that admit a Hurwitz group of automorphisms, and the AccolaMaclachlaclan and Wiman surfaces as we show in $\S 5$.

If $\mathcal{M}$ is a reflexible regular map, then the underlying Riemann surface $X=\mathcal{U} / K$ is symmetric and $K$ is normal in the NEC triangle group $\Gamma^{*}(2, m, n)$. However, if $X$ is symmetric, then $\mathcal{M}$ is not necessarily reflexible. If $m=n$, then by Lemma 2.1 there are two NEC groups which contain $\Gamma[2, m, m]$ with index 2 , namely $\Gamma^{*}(2, m, m)$ and the group of signature $(0 ;+;[m] ;\{(2)\})$. Let $\Gamma_{1}^{*}$ and $\Gamma_{2}^{*}$ be the NEC groups with the above signatures respectively. If $K$ is normal in $\Gamma_{2}^{*}$ and not in $\Gamma_{1}^{*}$, then $\mathcal{M}$ is not reflexible but $X$ is symmetric. However, if $m \neq n$, then $X$ is symmetric if and only if $\mathcal{M}$ is reflexible. See [23] for more details.

A map $\mathcal{M}$ of genus $g>1$ is said to be hyperelliptic if there is an automorphism $j$ of $\mathcal{M}$ such that $j^{2}=1$ and $\mathcal{M} /\langle j\rangle$ is isomorphic to a map on the sphere. The following theorem is given in [25].

Theorem 4.1 Let $\mathcal{M}$ be a regular map with underlying Riemann surface $X$. Then $\mathcal{M}$ is hyperelliptic if and only if $X$ is hyperelliptic.

If $\mathcal{M}$ is a hyperelliptic map of genus $g>1$, then $\mathcal{M}$ has an automorphism $j$ of order 2 such that $\mathcal{M} /\langle j\rangle$ is a map on the sphere. The automorphism $j$ is orientation-preserving and fixes $2 g+2$ points which can only be the vertices, the edge-centres or the face-centres of $\mathcal{M}$. Let $\mathcal{M}$ be of type $\{m, n\}$. If $j$ fixes only the face-centres of $\mathcal{M}$, then $\mathcal{M} /\langle j\rangle$ will be a regular map of type $\left\{m, \frac{n}{2}\right\}$ on the sphere. Note that unless $n$ is even $j$ cannot fix the face-centres of $\mathcal{M}$. Similarly, if $j$ fixes only the vertices of $\mathcal{M}$, then $\mathcal{M} /\langle j\rangle$ is a regular map of type $\left\{\frac{m}{2}, n\right\}$ on the sphere and in this case $m$ must be even.

We call a map on the sphere an m-star map if it consists of a single vertex $v$ and $m$ free edges incident with $v$. Let $\mathcal{M}^{\star}$ be an $m$-star map. 
Obviously, $\mathcal{M}^{\star}$ has a single face which can be regarded as an $m$-gon and $A u t^{+} \mathcal{M}^{\star}$ is isomorphic to $C_{m}$, the cyclic group of order $m$.

Let $\mathcal{M}$ be a hyperelliptic map of genus $g>1$ and $j: \mathcal{M} \rightarrow \mathcal{M}$ the hyperelliptic involution. If $\mathcal{M} /\langle j\rangle$ is an $m$-star map on the sphere for some $m$ then we call $\mathcal{M}$ a double-star map. Note that unless $j$ fixes the edgecentres of $\mathcal{M}, \mathcal{M} /\langle j\rangle$ is never an $m$-star map on the sphere.

\section{Accola-Maclachlan and Wiman surfaces}

Let $g>1$ be an integer and $\mu(g)$ be the maximum number of conformal automorphisms of all Riemann surfaces of genus $g$. Then it is known that $\mu(g) \leq 84(g-1)$ and this upper bound is attained for infinitely many $g>1$, see Macbeath [16]. If $X=\mathcal{U} / K$ is a Riemann surface of genus $g>1$ with $\left|A u t^{+} X\right|=84(g-1)$, then $A u t^{+} X$ is called a Hurwitz group and in this case $A u t^{+} X \simeq \Gamma / K$ and $\Gamma$ is a Fuchsian triangle group $\Gamma[2,3,7]$. It was shown independently by Accola [1] and Maclachlan [18] that $\mu(g) \geq 8(g+1)$ and for every $g \geq 2$ there is a Riemann surface of genus $g$ with $8(g+1)$ conformal automorphisms. These surfaces are known as Accola-Maclachlan surfaces.

The Accola-Maclachlan surfaces are constructed as follows. Consider the map on the Riemann sphere that has vertices at the $(2 g+2)$ th roots of 1 and edges joining these vertices along the equator. Such a map is called a regular dihedron. This is a regular map on the sphere with $2 g+2$ vertices, $2 g+2$ edges and 2 faces. Consider the two sheeted covering of this map with branch points at the vertices. We thus have a regular map with $2 g+2$ vertices, $4 g+4$ edges and 4 faces and by the Euler-Poincaré formula, the underlying surface has genus $g$. We call this map the Accola-Maclachlan map. The automorphism group of the above regular dihedron is isomorphic to $D_{2 g+2}$ and so has order $4 g+4$, and thus the number of automorphisms of the two-sheeted cover is $8 g+8$. The Riemann surface $X$ that underlies this map is the Accola-Maclachlan surface and we see that for every genus $g$ both this surface and map admit $8 g+8$ (conformal) automorphisms showing that the lower bound for $\mu(g)$ is always attained. As every vertex of the map has valency 4 , and every face is a $(2 g+2)$-gon the Accola-Machlachlan surface is a Platonic surface uniformised by a normal subgroup of index $8 g+8$ in the triangle group $\Gamma[2,4,2 g+2]$. If $R$ is the rotation of order 4 that fixes the $2 g+2$ vertices then by construction $R^{2}$ is the hyperelliptic involution and hence central in AutX. Thus we get the following presentation for the conformal automorphism group of $X$ and the Accola-Maclachlan map underlying $X$ :

$$
\left\langle R, S \mid R^{4}=S^{2 g+2}=(R S)^{2}=\left(R^{-1} S\right)^{2}=I\right\rangle .
$$


If we factor out $A u t^{+} X$ by the cyclic group of order 2 generated by $R^{2}$ we get $D_{2 g+2}$ as expected.

From the question "which Riemann surfaces admit automorphisms of the largest order" we get other classes of Platonic surfaces of interest to us. According to a classical theorem of Wiman [26] the largest possible order of an automorphism of a Riemann surface of genus $g$ is $4 g+2$ and the second largest possible order is $4 g$. (Also, see [10].) These are obtained as kernels of smooth homomorphisms of the triangle groups $\Gamma[2,2 g+1,4 g+2]$ and $\Gamma[2,4 g, 4 g]$ onto $C_{4 g+2}$ and $C_{4 g}$, respectively and thus these surfaces are Platonic. Following Kulkarni $[14,15]$ we call these surfaces Wiman surfaces of types $I$ and $I I$, respectively.

Let $X_{1}$ and $X_{2}$ be the Wiman surfaces of type $I$ and $I I$, respectively. Then $A u t^{+} X_{1} \simeq C_{4 g+2}$, where $g>1$ is the genus of $X_{1}$. However, $C_{4 g}$ is a subgroup of $A u t^{+} X_{2}$ with index 2. This comes from the inclusion relationship $\Gamma[2,4 g, 4 g]<\Gamma[2,4,4 g]$ of Lemma 2.2 and we can then show that $A u t^{+} X_{2}$ has a presentation

$$
\left\langle x, y \mid x^{2}=y^{4 g}=1, x y x^{-1}=y^{2 g-1}\right\rangle
$$

where $g>2$ is the genus of $X_{2}$. See Kulkarni [15].

\section{Double-star maps and the Wiman and Accola-Mac- lachlan surfaces}

In this section we shall see that the regular maps that are underlied by the Accola-Maclachlan and Wiman surfaces are double-star maps as defined at the end of $\S 4$. We first note the following facts. As the automorphism group of the $m$-star map is isomorphic to $C_{m}$ and as the hyperelliptic involution is central, the automorphism group of a double $m$-star map is isomorphic to either $C_{2} \times C_{m}$, or to $C_{2 m}$. Secondly, (and this applies to all two-sheeted covers) if a vertex of valency $k$ is the image of a branch point there are either two vertices of valency $k$, or, in the case when this vertex is the image of a branch point, one vertex of valency $2 k$. Thus, when we consider the case where the vertex is the image of a branch-point we go from smooth homomorphisms of $\Gamma[2, k, u] \rightarrow G$ to smooth homomorphisms of $\Gamma[2,2 k, u] \rightarrow \hat{G}$ where $|\hat{G}|=2|G|$. If the face-centre was also the image of a branch-point we would replace $u$ by $2 u$. Now if the edge-centres were images of branch-points then the period 2 would double to a period 4 and so the two-sheeted cover is no longer a map in the sense we have defined it here. (In some works the structure we have is called a hypermap, and, in others, a non-clean dessin). However a star map only has free edges and these could be described as 
edges of valency 1 . Formally, the group of $\Gamma[1, m, m]$ is isomorphic to $C_{m}$ and so a two-sheeted cover corresponds to a smooth image of $\Gamma[2, k, u]$ where $k, u$ are either $m$ or $2 m$, depending on whether there is branching over the vertex or face centre, as described above.

A double-star map on a surface of genus $g$ is the two-sheeted cover of an m-star map on the sphere. There are three possibilities (up to duality).

(i) There is only branching over the free end-points. As the number of branch points is equal to $2 g+2$ we have $m=2 g+2$. Thus we are considering smooth homomorphisms of $\Gamma[2,2 g+2,2 g+2]$ to either $C_{4 g+4}$ or to $C_{2} \times C_{2 g+2}$. However, it is easy to see that there is no smooth homomorphism in the first case, so we here have a doublestar map with automorphism group $C_{2} \times C_{2 g+2}$. As the corresponding Riemann surface is the two-sheeted cover of the sphere branched over the $(2 g+2)$ th roots of 1 , it is the Accola-Maclachlan surface. (Using Lemma 2.2, $\Gamma[2,2 g+2,2 g+2] \triangleleft \Gamma[2,4,2 g+2]$ with index 2 , and then we can obtain the description of the Accola-Maclachlan surface given in $\S 5$ in terms of the triangle group $\Gamma[2,4,2 g+2]$.)

(ii) There is branching over the free end-points and at the vertex. As above we see that $m=2 g+1$ and we are considering smooth homomorphisms from $\Gamma[2,2 g+1,4 g+2]$ to $C_{4 g+2}$, (Note that $C_{2} \times C_{2 g+1} \cong C_{4 g+2}$.) In this case the corresponding Riemann surface is the Wiman surface of type I. We get the same result by considering the dual situation of branching over the free end-points and the unique face centre.

(iii) There is branching over the free end-points, the vertex and the face center. Now $m=2 g$ and we are considering smooth homomorphisms from $\Gamma[2,4 g, 4 g]$ to either $C_{4 g}$ or to $C_{2} \times C_{2 g}$. As the latter group has no element of order $4 g$, the image must be $C_{4 g}$ and so the underlying Riemann surface of the double-star map is the Wiman surface of type II.

\section{The Medial Map}

Let $\mathcal{M}$ be a regular map of type $\{m, m\}$ lying on a surface of genus $g>1$ (so $m>4$ ). As each vertex of $\mathcal{M}$ has valency $m$ each angle at a vertex is equal to $2 \pi / m$. A medial of $\mathcal{M}$ is a geodesic arc whose end-points are at the edge-centres of adjacent edges of a face of $\mathcal{M}$ and whose interior lies inside the interior of that face and the medial map $\operatorname{Med}(\mathcal{M})$ of $\mathcal{M}$ has as vertices the edge-centres of $\mathcal{M}$, as edges the medials of $\mathcal{M}$. It has two kinds of faces; the first kind lie completely in the faces of $\mathcal{M}$, and their centre is at the corresponding face-centre. The second kind of face has as their 
centres the vertices of $\mathcal{M}$. As each angle at a vertex of $\mathcal{M}$ is $2 \pi / m$ all faces of $\operatorname{Med}(\mathcal{M})$ have size $m$. Thus if $\mathcal{M}$ has $|V|$ vertices, $|E|$ edges and $|F|$ faces then $\operatorname{Med}(\mathcal{M})$ has $|E|$ vertices, and $2|E|$ edges, (to see the latter we note that every vertex of $\operatorname{Med}(\mathcal{M})$ has valency 4 and each edge of $\mathcal{M}$ and a neighbouring edge give the same edge of $\operatorname{Med}(\mathcal{M})$ ). We now see that, as expected, the Euler characteristic of $\mathcal{M}$ and $\operatorname{Med}(\mathcal{M})$ are the same. Also, every vertex of $\operatorname{Med}(\mathcal{M})$ has valency 4 and every face has valency $m$, so that the $\operatorname{Med}(\mathcal{M})$ has type $\{4, m\}$. By Lemma 2.2, $\Gamma[2, m, m]<\Gamma[2,4, m]$ with index 2 , so that if $M<\Gamma[2, m, m]$ then $M$ is a map subgroup for a map $\mathcal{M}$ of type $\{m, m\}$ and also of a second map of type $\{4, m\}$. By considering fundamental regions it is easy to see that this second map is $\operatorname{Med}(\mathcal{M})$. (Note that it is not necessarily the case that $\operatorname{Med}(\mathcal{M})$ is regular. However from $[12], \S 6, \operatorname{Med}(\mathcal{M})$ is edge transitive and is regular if and only if there is an automorphism of order 2 that fixes the mid-point of an edge, and so fixes that edge setwise while reversing its direction.)

Theorem 7.1 The medials of a map $\mathcal{M}$ of type $\{m, m\}$ are mirrors of the underlying Riemann surface of $\mathcal{M}$.

Proof. Let $F$ be a face of $\mathcal{M}$, and $V$ be a vertex of $F$. We may suppose that $F$ is a regular hyperbolic polygon inside the unit disc with centre at the origin 0 . Let $V_{1}$ and $V_{2}$ be the two vertices adjacent to $V$ in $F$ and let $m$ be the medial joining the midpoints $P_{1}$ and $P_{2}$ of $V_{1} V$ and $V V_{2}$ respectively. Then the line $0 V$ intersects the medial $m$ orthogonally. Now the angles $V 0 P_{1}$ and $P_{1} V 0$ are both equal to $\pi / m$. It follows that reflection in the medial $m$ interchanges $V$ and 0 . Thus this reflection preserves the $\operatorname{map} \operatorname{Med}(\mathcal{M})$ and hence acts as a symmetry of the underlying Riemann surface of $\operatorname{Med}(\mathcal{M})$, which is the same as the underlying Riemann surface of $\mathcal{M}$ as by Lemma 2.2 we have the inclusion relationship $\Gamma[2, m, m]<\Gamma[2,4, m]$.

Corollary 7.1 A Platonic Riemann surface that underlies a regular map of type $\{m, m\}$ is symmetric.

(This was noted in [23] in the case $m=7$.)

Lemma 7.1 Let $X$ be a Platonic Riemann surface that underlies a regular map $\mathcal{M}$ of type $\{m, m\}$ and suppose that $A u t^{+} \mathcal{M}$ is abelian. Then $m$ is even and

(i) If $m$ is divisible by 4 (say $m=4 g$ ) then $X$ is the Wiman surface of type II of genus $g$ and $A u t^{+} \mathcal{M} \cong C_{4 g}$.

(ii) If $m \equiv 2 \bmod 4$ (say $m=2 g+2$ ) then $X$ is the Accola-Maclachlan surface of genus $g$ and $A u t^{+} \mathcal{M} \cong C_{2} \times C_{2 g+2}$. 
Proof. If $m$ is odd then by abelianising the triangle group $\Gamma[2, m, m]$ we find that there is no smooth homomorphism onto an abelian group. Thus $m$ is even. If $m=4 g$ then the abelianized group is $C_{4 g}$ generated by $T$ say and if $\Gamma[2, m, m]$ has presentation $\left\langle x, y, z \mid x^{m}=y^{m}=z^{2}=x y z=1\right\rangle$ then $x \mapsto T, y \mapsto T^{(m / 2)-1}, z \mapsto T^{m / 2}$ extends to a smooth homomorphism from $\Gamma[2, m, m]$ onto $C_{4 g}$. If $m=2 g+2$ and if $C_{2}$ is generated by $U$ and $C_{2 g+2}$ is generated by $T$ then $x \mapsto(1, T), y \mapsto\left(U, T^{-1}\right), z \mapsto(U, 1)$ extends to a smooth homomorphism from $\Gamma[2, m, m]$ onto $C_{2} \times C_{2 g+2}$.

In Figure 2 we draw the fundamental domain for the group that represents the Wiman surface of type II and genus 3. The fundamental domain is a regular hyperbolic $4 g$-gon which is a union of $4 g$ triangles with an angle of $\pi / 2 g$ at the centre and angles equal to $\pi / 4 g$ at the vertices. In Figure 3 we draw the fundamental domain for the group that represents the Accola-Maclachlan surface of genus 2. In both cases the side pairings are as indicated. (These regions and their side-pairings follow from the Reidemeister-Schreier method as described, for example in [11].) Also, on these diagrams we draw the medial graphs whose edges, by Theorem 7.1, project to mirrors of the underlying surfaces.

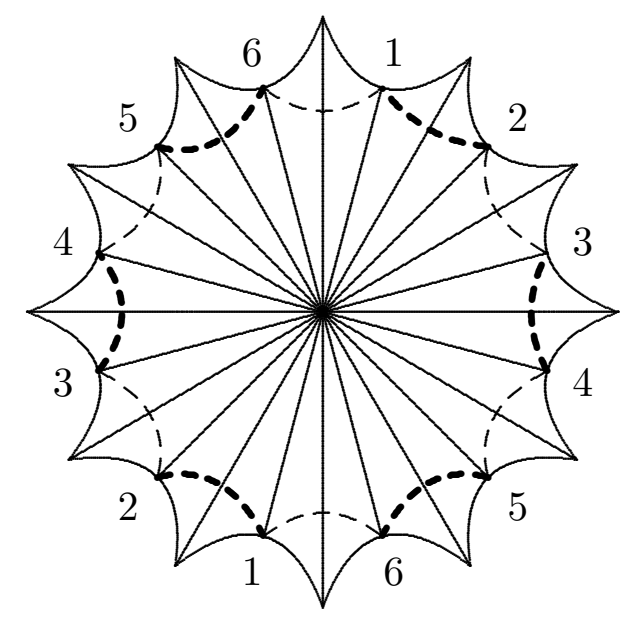

Figure 2

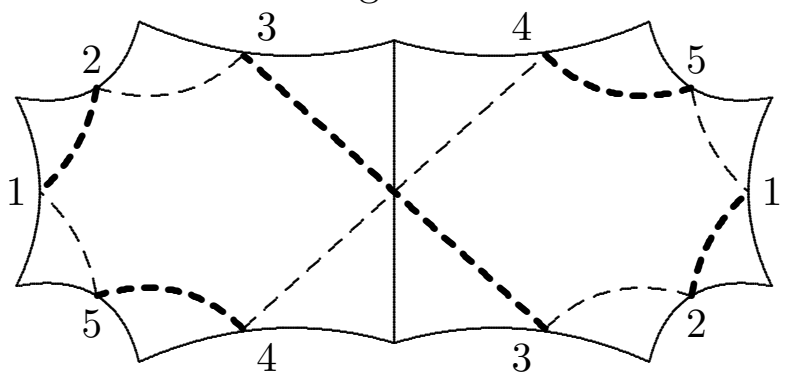

Figure 3 
If we consider the Wiman surface of type II we see that we have $4 g$ medial edges on the polygon, which after identification become $2 g$ mirrors on the surface. We form the Accola-Maclachlan surface from two adjacent regular $(2 g+2)$-gons. These contain a total of $4 g+4$ medial edges which after identification (possibly after continuing an edge from one polygon to another) become $2 g+2$ mirrors on the surface. We now show that, in each case, half of the mirrors are axes of one of the symmetries, and the other half are axes for the other symmetry. To prove this we just need the following.

Lemma 7.2 Let $W$ be a reflection of the Wiman surface of type II or the Accola-Maclachlan surface and let $T$ be the conformal automorphism of order $4 g$ or $2 g+2$ respectively. If $W$ fixes some medials then it commutes with $T^{2}$.

Proof. When we lift the extended group generated by the conformal automorphisms and $W$ to the upper-half plane $\mathcal{U}$ we obtain the NEC group $\Delta$ with signature $(0 ;+;[m] ;\{(2)\})$ and presentation

$$
\left\langle x, c \mid x^{m}=c^{2}=\left(c x^{-1} c x\right)^{2}=1\right\rangle
$$

where the homomorphism from $\Delta$ to Aut $\mathcal{M}$ is just $c \mapsto W$ and $x \mapsto T$. Now $T, W T W^{-1} \in A u t^{+} \mathcal{M}$ and as $A u t^{+} \mathcal{M}$ is abelian, and $W$ has order 2

$$
T W T W=W T W T \text {. }
$$

Also, as $c x^{-1} c x$ is self-inverse in $\Delta$ we also have

$$
W T^{-1} W T=T^{-1} W T W .
$$

Thus $T W T W W T^{-1} W T=W T W T T^{-1} W T W$ and this gives $T^{2} W=W T^{2}$ as required.

Note that if $W$ fixes no medials then the above argument does not apply. This is because in this case we obtain the NEC triangle group $\Gamma^{*}(2, m, m)$, which is generated by reflections. However, the extended group cannot be generated by symmetries and so there is no admissible epimorphism between them.

As a consequence we have a geometric proof of a known result.

Theorem 7.2 The Accola-Maclachlan surface is an M-surface. The Wiman surface of type II is an (M-1)-surface.

Proof. Consider the Accola-Maclachlan surface; as observed before Lemma 7.2 , this contains $2 g+2$ mirrors coming from the medial edges after identifications of the edges. If $m$ is one of these mirrors fixed by a symmetry 
$W$, say, and if $T$ is the conformal automorphism of order $2 g+2$ of the surface then $W T^{2 j}(m)=T^{2 j} W(m)=T^{2 j}(m)$, for $j=0, \ldots, g$, showing that $W$ has $g+1$ mirrors as claimed. Thus the Accola-Maclachlan surfaces are M-surfaces and a similar proof applies to the Wiman surface of type II.

We also note the known result that the Wiman surfaces of type I admit two non-conjugate symmetries each fixing one mirror. To see this just observe that the Wiman surface of type I of genus $g$ can be formed by identifying the opposite edges of a regular hyperbolic $(4 g+2)$-gon. (See $[14,15])$. If this surface is denoted by $X_{1}$ then $A u t^{+} X_{1} \cong C_{4 g+2}$ and $A u t X_{1} \cong D_{4 g+2}$. The symmetries are the two classes of involutions outside the cyclic subgroup of order 2. Each fixes either a single mirror coming (after identification of sides) from a diagonal line of the polygon or from a perpendicular bisector of a pair of opposite edges.

\section{Platonic $\mathrm{M}$ and (M-1)-surfaces}

Theorem 8.1 Platonic $M$ and (M-1)-surfaces are hyperelliptic.

Proof. We shall assume that $X=\mathcal{U} / K$ is a Platonic M-surface which is non-hyperelliptic and obtain a contradiction. As $X$ is Platonic, $K$ is normal in a triangle group $\Gamma[2, m, n]$, where $1 / m+1 / n<1 / 2$. If $m \neq n$, then as $X$ is symmetric, $K$ is also normal in the NEC triangle group $\Gamma^{*}(2, m, n)$ and $A u t X$ can be generated by three reflective symmetries $A, B, C$ obeying

$$
A^{2}=B^{2}=C^{2}=(A B)^{2}=(B C)^{m}=(C A)^{n}=I .
$$

This group lifts to an NEC triangle group $\Delta$ with presentation (4.1). The M-symmetry lifts to a reflection in $\Gamma^{*}(2, m, n)$ and as every reflection in $\Delta$ is conjugate to one of $a, b, c$ it follows that at least one of $A, B, C$ is the Msymmetry. Now if $A$ is the M-symmetry, then by Theorem $3.2 A$ is central in $A u t X$. So $n=2$ which is a contradiction as $1 / m+1 / n<1 / 2$ and we get similar contradictions if we choose $B$ or $C$ is the M-symmetry.

If $m=n$, then there is another NEC group $\left(\Gamma^{\prime}\right)^{*}$ which contains $\Gamma[2, m, m]$ with index 2 and has signature

$$
(0 ;+;[m] ;\{(2)\})
$$

$\left(\Gamma^{\prime}\right)^{*}$ has a presentation

$$
\left\langle x, c \mid x^{m}=c^{2}=\left(c x c x^{-1}\right)^{2}=1\right\rangle .
$$

However, as we shall see now, there is no admissible epimorphism from $\left(\Gamma^{\prime}\right)^{*}$ to AutX. Assume that $\theta:\left(\Gamma^{\prime}\right)^{*} \rightarrow A u t X$ is an admissible epimorphism. By 
Theorem 3.2 AutX is isomorphic to $C_{2} \times A u t^{+} X$ and $C_{2}$ is generated by the unique M-symmetry. It follows from (8.1) that all reflections of $\left(\Gamma^{\prime}\right)^{*}$ are conjugate and therefore their images under $\theta$ must be conjugate in Aut $X$. The reflection generators of $\left(\Gamma^{\prime}\right)^{*}$ must be mapped to reflective symmetries of $X$ by $\theta$ and as every reflection in $\left(\Gamma^{\prime}\right)^{*}$ is conjugate to $c$, it follows that $\theta(c)$ is the unique M-symmetry which is central. Then $c x c x^{-1}$ will be in $\operatorname{Ker} \theta$, the kernel of $\theta$, which is a contradiction as $\operatorname{Ker} \theta$ must be torsion-free. Thus, every Platonic M-surface is hyperelliptic and using Theorem 3.3 (and the fact that every Riemann surface of genus 2 is hyperelliptic) we can show that every Platonic (M-1)-surface is hyperelliptic in the same way.

Lemma 8.1 Let $X$ be an M-surface of genus $g>1$ and $T: X \rightarrow X$ be an $M$-symmetry. If $X$ admits another symmetry $S$, then $T S$ has order 2 or 4 . If $g>2$ then the same result applies to $(M-1)$-symmetries acting on an (M-1)-surface.

Proof. Let $X$ be an M-surface. If $X$ is non-hyperelliptic, then by Theorem 3.2, $T$ is the unique M-symmetry, which is central in AutX, and so $T S=S T$. Therefore, $T S$ has order 2. If $X$ is hyperelliptic and $S$ and $T$ commute, then TS has order 2. If $S$ and $T$ do not commute, then $S T S$ is conjugate to $T$ and is the other M-symmetry. By [5] their product TSTS is the hyperelliptic involution and hence $T S$ has order 4 . This has also been proved in [6], (Corollary 2 of Theorem 6). Similarly, we can show that if $X$ is an (M-1)-surface of genus $g>2$ then $S T$ has order 2 or 4 .

Theorem 8.2 For every $g>1$ the Accola-Maclachlan surface is the only Platonic M-surface of genus $g$.

Proof. Suppose that $X=\mathcal{U} / K$ is a Platonic M-surface of genus $g>1$. Then $K$ is normal in a triangle group $\Gamma=\Gamma[2, m, n]$, where $1 / m+1 / n<1 / 2$. This means $X$ carries a regular map $\mathcal{M}$ of type $\{m, n\}$. If $m \neq n$, then as $X$ is symmetric, $K$ is also normal in the NEC triangle group $\Gamma^{*}(2, m, n)$ and Aut $X$ can be generated by three reflective symmetries $A, B, C$ obeying

$$
A^{2}=B^{2}=C^{2}=(A B)^{2}=(B C)^{m}=(A C)^{n}=I .
$$

It follows from Theorem 8.1 that $X$ is hyperelliptic and so admits two Msymmetries. As in the proof of Theorem 8.1, we can choose one of the generators of $A u t X$, for instance $B$, as an M-symmetry. It follows from Lemma 8.1 that $m$ is either 2 or 4 . Since $1 / m+1 / n<1 / 2, m$ cannot be 2 . So $m=4$ and $\mathcal{M}$ has type $\{4, n\}$. This means every face of $\mathcal{M}$ is surrounded by $n$ edges and each vertex has valency 4 . Now we want to find $n$. We can 
divide each face of $\mathcal{M}$ into $2 n$ triangles such that each triangle has interior angles $\pi / 2, \pi / 4$ and $\pi / n$. It follows from Lemma 8.1 that one side of each triangle is a part of a mirror of an M-symmetry. This is the side opposite to the angle $\pi / n$. We can observe that every edge of $\mathcal{M}$ lies on a mirror of an M-symmetry and the M-symmetries do not fix interior points of the faces of $\mathcal{M}$. Also, we can see that every vertex of $\mathcal{M}$ is a fixed-point of $J$, the hyperelliptic involution, and $J$ maps each edge of $\mathcal{M}$ to another edge such that these two edges form a mirror for an M-symmetry. The M-symmetries of $X$ have $2 g+2$ mirrors in total and hence $\mathcal{M}$ has $4 g+4$ edges. So the order of $A u t^{+} \mathcal{M}$ is $8 g+8$ and $\mathcal{M}$ has $\frac{8 g+8}{4}=2 g+2$ vertices. By the EulerPoincaré formula we find that $\mathcal{M}$ has 4 faces and so $n=\frac{8 g+8}{4}=2 g+2$. Therefore, $\mathcal{M}$ is of type $\{4,2 g+2\}$ and $\Gamma=\Gamma[2,4,2 g+2]$. Thus, $A u t^{+} \mathcal{M}$ can be generated by two automorphisms $R, S$ obeying

$$
R^{4}=S^{2 g+2}=(R S)^{2}=I .
$$

Here $R$ is a rotation about a vertex and we can see that $R^{2}$ is the hyperelliptic involution. Thus, $A u t^{+} \mathcal{M}$ is isomorphic to the group with presentation (5.1) and therefore $X$ is the Accola-Maclachlan surface of genus $g$.

Now we suppose that $m=n$. Then either $K$ is normal in $\Gamma^{*}(2, m, m)$ as above and the previous proof holds or $K \triangleleft \Delta$, where $\Delta$ is an NEC group of signature $(0 ;+;[m] ;\{(2)\})$ with a presentation $\left\langle x, c \mid x^{m}=c^{2}=\left(c x c x^{-1}\right)^{2}=1\right\rangle$ (See $\S 2$ and Lemma 2.1). Suppose that in the canonical homomorphism from $\Delta$ to $\Delta / K x \mapsto A$ and $c \mapsto C$. Now, by Theorem 3.2, the surface $X$ admits two M-symmetries whose product is the hyperelliptic involution and hence of order 2 . Thus, by the smoothness of the homomorphism from $\Delta$ to $\Delta / K$, we deduce that $\Delta$ must contain a subgroup isomorphic to $D_{2}$ and we may assume this is the group generated by $c$ and $x c x^{-1}$. Thus the two symmetries are $C$ and $A C A^{-1}$ and the hyperelliptic involution is $A C A^{-1} C$, which is central. Let $B=C A C$. Then $A u t^{+} X$ is generated by $A$ and $B$ obeying the relations $A^{m}=B^{m}=\left(A B^{-1}\right)^{2}=I$ with $A B^{-1}$ central. In particular, $A B^{-1}$ commutes with $B$ and so $A$ and $B$ commute and $A u t^{+} X$ is abelian. Thus $A^{2}=B^{2}$ and if $m$ is odd $A=B$ and $x c x^{-1} c$ belongs to the kernel of the homomorphism from $\Delta$ to $\Delta / K$. This contradicts the smoothness of this homomorphism and so $m$ is even and by Lemma 7.1, $X$ is the Accola-Maclachlan or Wiman surface of type II. However by [5] a Riemann surface of genus $g$ cannot admit two symmetries one fixing $g+1$ curves and the other fixing $g$ curves and so $X$ is the Accola-Maclachlan surface of genus $g$.

Theorem 8.3 For every $g>1$ the Wiman surface of type II is the only Platonic (M-1)-surface of genus $g$. 
Proof. For $g>2$ the proof is the same as that of Theorem 8.2. For $g=2$ this does not quite work as Theorem 3.3 and Lemma 8.1 do not hold in this case. However, for $g=2$ there are only 3 Platonic surfaces as can be shown by examining lists of Riemann surfaces of low genus admitting large automorphism groups, (e.g. see [4]). The three Platonic surfaces of genus 2 have full conformal automorphism groups equal to $C_{10}$, a group of order 24, or a group of order 48 (isomorphic to GL(2,3)); these come as smooth images of $\Gamma[2,5,10], \Gamma[2,4,6]$, or $\Gamma[2,3,8]$, respectively. We recognise the first surface as the Wiman surface of type I which we saw at the end of the previous paragraph only has symmetries fixing one mirror. The second is the Accola-Maclachlan surface of genus 2 which, as it admits a symmetry fixing 3 mirrors, cannot also have a symmetry fixing 2 mirrors. The final surface admits an automorphism of order 8 and so must be the Wiman surface of type II (which also follows from the inclusion relationship $\Gamma[2,8,8]<$ $\Gamma[2,3,8])$. Thus the Wiman surface of genus 2 is the unique Platonic (M-1)surface of genus 2 .

The $\mathrm{M}$ and ( $\mathrm{M}-1)$-surfaces we encountered in this paper were hyperelliptic. A hyperelliptic M-surface of genus $g>1$ corresponds to a complex algebraic curve

$$
C=\left\{(z, w) \in \mathbb{C}^{2} \mid w^{2}=\left(z-a_{1}\right)\left(z-a_{2}\right) \ldots\left(z-a_{2 g+2}\right)\right\},
$$

where each $a_{i} \in \mathbb{R}$ and $a_{i} \neq a_{j}$. Here the map $h:(z, w) \mapsto(z,-w)$ is the hyperelliptic involution, which fixes the points $\left(a_{1}, 0\right), \ldots,\left(a_{2 g+2}, 0\right)$ on $C$. The maps $s_{1}:(z, w) \mapsto(\bar{z}, \bar{w})$ and $s_{2}:(z, w) \mapsto(\bar{z},-\bar{w})$ are the M-symmetries. Similarly, a hyperelliptic (M-1)-surface of genus $g>1$ is represented by a curve

$$
C^{\prime}=\left\{(z, w) \in \mathbb{C}^{2} \mid w^{2}=\left(z-b_{1}\right)\left(z-b_{2}\right) \ldots\left(z-b_{2 g+2}\right)\right\},
$$

where two of the numbers $b_{i}$ are non-real and the others are real, and $b_{i} \neq b_{j}$. In this case, the hyperelliptic involution and the (M-1)-symmetries are given by the same formulae as above.

Similar work on symmetries of Accola-Maclachlan surfaces has been done by [2] and [7].

\section{References}

[1] Accola, R. D. M.: On the number of automorphisms of a closed Riemann surface. Trans. Amer. Math. Soc. 131 (1968), 398-408.

[2] Broughton, S. A., Bujalance, E., Costa, A. F., Gamboa, J. M. And Gromadzki, G.: Symmetries of Accola-Maclachlan and Kulkarni surfaces. Proc. Amer. Math. Soc. 127 (1999), no. 3, 637-646. 
[3] Bujalance, E., Etayo, J. J., Gamboa, J. M. and Gromadzki, G.: Automorphism groups of compact bordered Klein surfaces. A combinatorial approach. Lecture Notes in Mathematics 1439. Springer-Verlag, Berlin, 1990.

[4] Bujalance, E. and Singerman, D.: The symmetry type of a Riemann surface. Proc. London Math. Soc. (3) 51 (1985), 501-519.

[5] Bujalance, E. And Costa, A. F.: A combinatorial approach to the symmetries of $M$ and $M-1$ Riemann surfaces. In Discrete Groups and Geometry (Birmingham, 1991), 16-25. London Math. Soc. Lecture Note Ser. 173. Cambridge Univ. Press, Cambridge, 1992.

[6] Bujalance, E., Costa, A.F. And Singerman, D.: Application of Hoare's theorem to symmetries of Riemann surfaces. Ann. Acad. Sci. Fenn. Ser. A I Math. 18 (1993), 307-322.

[7] Cirre, F. J. and GamboA, J. M.: Compact Klein surfaces and real algebraic curves. In Topics on Riemann surfaces and Fuchsian groups (Madrid, 1998), 113-131. London Math. Soc. Lecture Note Ser. 287. Cambridge Univ. Press, Cambridge, 2001.

[8] Coxeter, H.S. M. and Moser, W. O. J.: Generators and relations for discrete groups. Fourth edition. Results in Mathematics and Related Areas 14. Springer-Verlag, Berlin-New York, 1980.

[9] Harnack, A.: Über die Vieltheiligkeit der ebenen algebraischen Curven. Math. Ann. 10 (1876), 189-198.

[10] Harvey, W. J.: Cyclic groups of automorphisms of a compact Riemann surface. Quart. J. Math. Oxford. Ser. (2) 17 (1966), 86-97.

[11] Hoare, A.H. M. and Singerman, D.: The orientability of subgroups of plane groups. In Groups-St. Andrews 1981 (St. Andrews 1981), 221-227. London Math. Soc. Lecture Note Ser. 71. Cambridge Univ. Press, Cambridge, 1982.

[12] Jones, G. A. And Singerman, D.: Theory of maps on orientable surfaces. Proc. London Math. Soc. (3) 37 (1978), 273-307.

[13] KLEIN, F.: On Riemann's theory of algebraic functions and their integrals. A supplement to the usual treatises. Dover Publications, New York, 1963.

[14] Kulkarni, R. S.: A note on Wiman and Accola-Maclachlan surfaces. Ann. Acad. Sci. Fenn. Ser. A I Math. 16 (1991), 83-94.

[15] KulKarni, R. S.: Riemann surfaces admitting large automorphism groups. In Extremal Riemann surfaces (San Francisco, CA, 1995), 63-79. Contemp. Math. 201. Amer. Math. Soc., Providence, RI, 1997.

[16] Macbeath, A. M.: On a theorem of Hurwitz. Proc. Glasgow Math. Assoc. 5 (1961), 90-96.

[17] Macbeath, A. M.: The classification of non-euclidean plane crystallographic groups. Canad. J. Math. 19 (1967), 1192-1205. 
[18] Maclachlan, C.: A bound for the number of automorphisms of a compact Riemann surface. J. London Math. Soc. 44 (1969), 265-272.

[19] Natanzon, S. M.: Automorphisms of the Riemann surface of an $M$-curve. Funct. Anal. Appl. 12 (1978) 228-229.

[20] Natanzon, S. M.: Automorphisms and real forms of a class of complex algebraic curves. Funct. Anal. Appl. 13 (1979), 148-150.

[21] Natanzon, S. M.: Lobachevskien geometry and automorphisms of complex $M$-curves. Selecta Math. Sovietica. 1 (1981), 81-99.

[22] Singerman, D.: Finitely maximal Fuchsian groups. J. London Math. Soc. (2) 6 (1972), 29-38.

[23] Singerman, D.: Symmetries of Riemann surfaces with large automorphism group. Math. Ann. 210 (1974), 17-32.

[24] Singerman, D.: On the structure of non-Euclidean crystallographic groups. Proc. Cambridge Philos. Soc. 76 (1974), 233-240.

[25] Singerman, D.: Hyperelliptic maps and surfaces. Math. Slovaca 47 (1997), 93-97.

[26] Wiman, A.: Über die hyperelliptischen curven und diejenigen vom geschlechte $p=3$ welche eindeutigen transformationen in sich zulassen. Bihang Till Kongl. Svenska Veienskaps-Akademiens Handlingar, (Stockholm 1895-6), bd.21, 1-23.

Recibido: 10 de mayo de 2007

Revisado: 15 de octubre de 2007

Adnan Melekoğlu Department of Mathematics Faculty of Arts and Sciences Adnan Menderes University 09010 Aydin, Turkey amelekoglu@adu.edu.tr

David Singerman School of Mathematics University of Southampton Southampton, SO17 1BJ, England

d.singerman@soton.ac.uk 